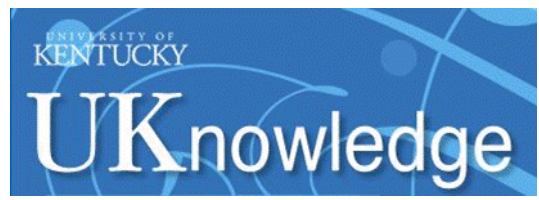

University of Kentucky

UKnowledge

Biosystems and Agricultural Engineering Faculty Publications

2018

\title{
Application of Acoustic Emission and Machine Learning to Detect Codling Moth Infested Apples
}

\author{
Mengxing Li \\ University of Kentucky \\ Nader Ekramirad \\ University of Kentucky \\ Ahmed Rady \\ University of Kentucky
}

Akinbode A. Adedeji

University of Kentucky, akinbode.adedeji@uky.edu

Follow this and additional works at: https://uknowledge.uky.edu/bae_facpub

Part of the Bioresource and Agricultural Engineering Commons, Computer Sciences Commons, and the Plant Sciences Commons

Right click to open a feedback form in a new tab to let us know how this document benefits you.

\section{Repository Citation}

Li, Mengxing; Ekramirad, Nader; Rady, Ahmed; and Adedeji, Akinbode A., "Application of Acoustic Emission and Machine Learning to Detect Codling Moth Infested Apples" (2018). Biosystems and Agricultural Engineering Faculty Publications. 224.

https://uknowledge.uky.edu/bae_facpub/224

This Article is brought to you for free and open access by the Biosystems and Agricultural Engineering at UKnowledge. It has been accepted for inclusion in Biosystems and Agricultural Engineering Faculty Publications by an authorized administrator of UKnowledge. For more information, please contact UKnowledge@lsv.uky.edu. 


\section{Application of Acoustic Emission and Machine Learning to Detect Codling Moth Infested Apples}

\section{Digital Object Identifier (DOI)}

https://doi.org/10.13031/trans.12548

\section{Notes/Citation Information}

Published in Transactions of the ASABE, v. 61, issue 3, p. 1157-1164.

(C) 2018 American Society of Agricultural and Biological Engineers

The copyright holder has granted the permission for posting the article here. 


\title{
APPLICATION OF ACOUSTIC EMISSION AND MACHINE LEARNING TO DETECT CODLING MOTH INFESTED APPLES
}

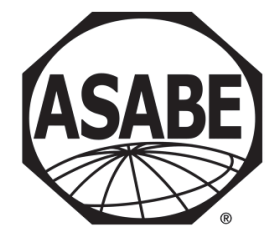

\author{
M. Li, N. Ekramirad, A. Rady, A. Adedeji
}

\begin{abstract}
Incidence of codling moth (CM) (Cydia pomonella L.) infestation in apples has been a major concern in North America for decades. CM larvae bore deep into the fruit, making it unmarketable. An effective noninvasive method to detect larvae-infested apples is necessary to ensure that apples are CM-free in post-harvest processing. In this study, a novel approach using an acoustic emission (AE) system and subsequent machine learning methods was applied to classify larvaeinfested apples from intact apples. 'GoldRush' apples were infested with CM neonates and stored at the same conditions as intact apples. The AE system was used to collect the data emitted by 80 larvae-infested and intact apples in total. Eleven AE features that changed with signaling time were obtained with the AE system. For each feature, the area under the curve along the signaling time was calculated and used as an independent input variable for the machine learning algorithms, which included linear discriminant analysis (LDA) and ensemble method adaptive boosting. With signaling times ranging from 0.5 to $120 \mathrm{~s}$, classification rates for infested versus intact apples ranged from $91 \%$ to $100 \%$ for the training set and from $83 \%$ to $100 \%$ for the test set. The quick signal collection and high classification accuracy obtained in this study show the potential of AE for detecting and classifying CM-infested apples.
\end{abstract}

Keywords. Acoustic emission, Apple, Codling moth, Machine learning, Pest infestation.

$\mathrm{A}$ pple is one of the most important fruits in the world and has its origin in central Asia and Europe (Konopacka et al., 2010). The U.S. is the largest exporter of apples in the world, even though the U.S. is the third largest producer (Ivanovic and Jelocnik, 2017). The main countries that import U.S. apples, namely Taiwan, China, Japan, Thailand, and India, have zero tolerance for any form of pest infestation (Walker et al., 2013). When just one infested apple is detected, the entire shipment is liable to rejection. Just three incidents of infestation detected in a single year in apples imported to Taiwan will lead to a ban from the source country. Codling moth (CM) (Cydia pomonella L.) is a serious insect pest in apples. CM infestation of apples, and subsequent microbial contamination, severely damages the quality of apples. The damage and economic loss due to $\mathrm{CM}$ infestation are usually enormous (Dorn and Piñero, 2009). CM usually lays eggs on the calyx or stem of the apple. The eggs become larvae, and the larvae burrow into the apple, where they continue to grow. Because the larvae enter through the calyx, the outer surfaces of infested apples show no changes, which presents a chal-

Submitted for review in July 2017 as manuscript number PRS 12548; approved for publication by the Processing Systems Community of ASABE in January 2018.

The authors are Mengxing Li, Graduate Student, Nader Ekramirad, Graduate Student, Ahmed Rady, Post-Doctoral Fellow, and Akinbode Adedeji, Assistant Professor, Department of Biosystems and Agricultural Engineering, University of Kentucky, Lexington, Kentucky. Corresponding author: Akinbode Adedeji, 128 C.E. Barnhart Building, University of Kentucky, Lexington, KY 40546; phone: 859-218-4355; e-mail: akinbode.adedeji@uky.edu. lenging task for apple sorting and grading.

Conventional methods in the industry for detecting CMinfested apples are random selection, cutting, and inspection of the apples manually, which is destructive, laborious, and increases the logistic cost (Mohana et al., 2013). This is coupled with the fact that not all of the apples are inspected. Nondestructive methods have been described for detecting defects and quality attributes in fruits and vegetables, such as x-ray imaging for apple defect detection (Schatzki et al., 1997), hyperspectral imaging for CM infestation detection (Rady et al., 2017), and magnetic resonance imaging for prediction of tomato quality attributes and mechanical damage (Milczarek et al., 2009; Zhang and McCarthy, 2012). Each of these methods has limitations, such as the inability to detect changes beyond the surface reflectance of a thick object (such as an apple) with hyperspectral imaging, and the response time of thermal imaging, which may not be practical for online applications. In this article, we propose a novel nondestructive method that can distinguish CM-infested apples from intact apples by delineating the acoustic emission differences in the apples.

Acoustic emission (AE) refers to the generation and radiation of acoustic (elastic) waves in solids that occur when a material undergoes irreversible changes in its internal structure (Muravin, 2009). AE measurements have been applied to measure events as diverse as microcracking in metals, earthquake tremors, chemical reactions, and microbubble processes (Stencel et al., 2009). The AE device available in the Food Engineering Laboratory of the Department of Biosystems and Agricultural Engineering at the University of Kentucky is capable of recording signals every $0.2 \mathrm{~s}$, thereby 
producing large amounts of data, which means that much more information can be acquired than with most conventional AE devices. This device has successfully detected the signals produced by Escherichia coli and Lactococcus latic, ssp. lactis during the growth phase. Ghosh et al. (2013) proposed that the same AE system can provide real-time data on the metabolic activity of $L$. latic ssp. lactis and was able to dynamically monitor phase infection of cells. Yang et al. (2014) reported the establishment of a relationship between crop AE and disease stress, which allowed distinguishing diseased crops from healthy crops. Some previous studies showed that an acoustic device could be optimized to predict watermelon firmness (Mao et al., 2016) and classify extruded bread with different water activities (Swietlicka et al., 2015), and a contact AE detector was applied to evaluate apple texture with mechanical destruction of apples (Zdunek et al., 2011).

However, most previous AE studies have measured food quality attributes by mechanically destroying the food. In this study, we propose that AE can distinguish CM-infested apples from intact apples nondestructively. From the multiple AE parameters generated, machine learning methods are needed to classify larvae-infested apples from intact apples. Linear discriminant analysis (LDA) and boosting are two commonly used machine learning methods. LDA is a probabilistic parametric classification technique for finding linear discriminants (LD), which are linear combinations of the original variables that maximize the variance between categories and minimize the variance within categories. LDA is commonly used to separate two or more classes of objects. Gorji-Chakespari et al. (2016) and Xu et al. (2016) used LDA to classify essential oils and edible oil oxidation with an electronic nose, respectively. Boosting is a tree-based ensemble method that combines weak learners to build a strong classifier. Boosting consists of iteratively learning weak classifiers with respect to a distribution and adding them to a final strong classifier. The goal of boosting is to assign higher weights to classifiers with high accuracy during the training process, while decreasing the weight of classifiers with lower accuracy, which leads to an increasing probability of a correct final output for the ensemble (Mohareb et al., 2016). Li et al. (2015) applied adaptive boosting and increased the accuracy for detecting total volatile basic nitrogen content in pork. Dai et al. (2015) used adaptive boosting to enhance the visible/hyperspectral data analysis for rapid detection of frozen and unfrozen prawns.

This study pioneers the application of AE for nondestructive detection of live insect larvae in fruits like apple. To the best of our knowledge, no information is available in the literature on AE signal transmission and analysis for apples with live insects. The objective of this study is therefore to test the feasibility of AE signal collection for apple classification and to construct statistical models to assess the classification performance.

\section{Materials And Methods APPLES}

'GoldRush' apples were purchased from Evans Orchard \& Cider Mill (Georgetown, Ky.) at two different times. The apples were harvested in fall 2015 and stored in a $4^{\circ} \mathrm{C}$ room on the farm. The purchased apples were transported to the lab and washed with soap and water to remove pesticide residue that could prevent the growth of CM. After washing, the apples were air-dried overnight at room temperature (around $24^{\circ} \mathrm{C}$ ). The cleaned apples were then stored in a cardboard box in a $4^{\circ} \mathrm{C}$ room for one week before inoculation with CM eggs.

\section{INOCULATION}

The CM life cycle is illustrated in figure 1 . The life stages of $\mathrm{CM}$ at $28^{\circ} \mathrm{C}$ include egg (around 4 days), larva (16 days), pupa ( 7 days), and adult (10 days). Hatching of eggs to release the neonates (newly hatched larvae) requires high hu-

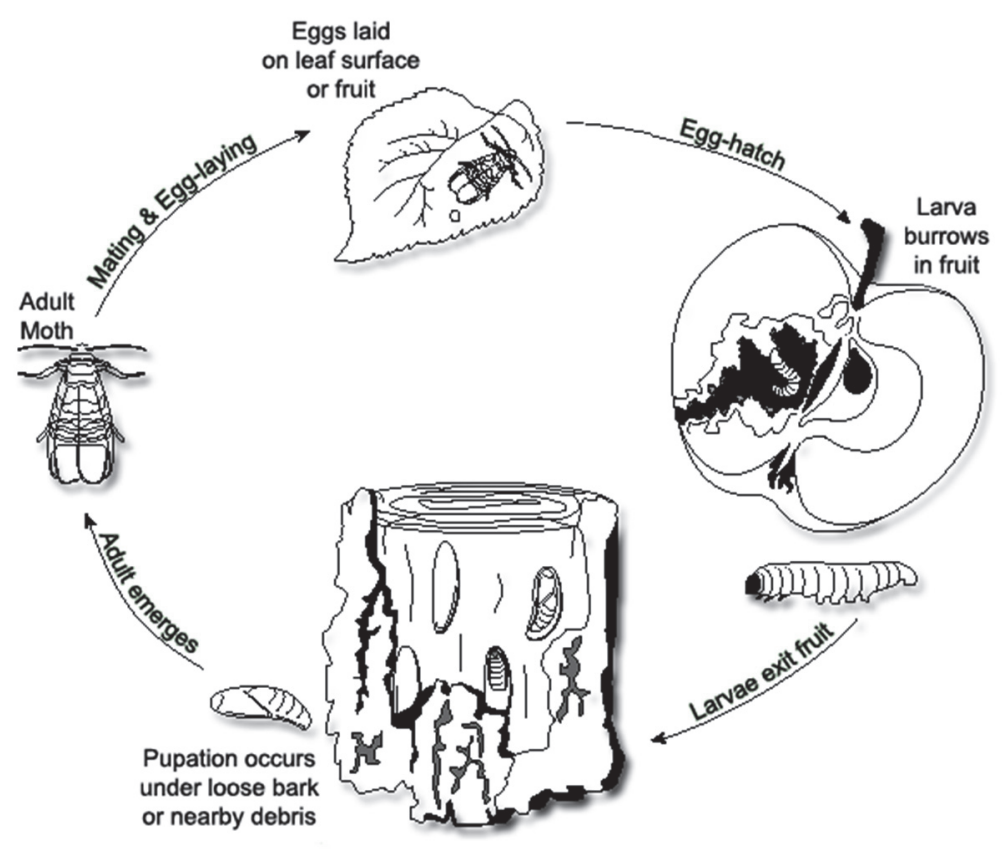

Figure 1. CM life cycle (source: http://www.oksir.org/lifecycle.asp). 
midity. After hatching, 50\% humidity is enough to keep moisture off the plant surfaces so that the neonates do not get stuck and essentially drown. The purpose of this experiment was to mimic the conditions in which neonates infest apples, and then use the CM-infested apples for the AE study.

$\mathrm{CM}$ eggs were purchased from Benzon Research (Carlisle, $\mathrm{Pa}$.). When the eggs were received, they were placed in a $928 \mathrm{~mL}$ (32 oz) polypropylene container with a slightly dampened cotton ball at the bottom. This container was closed and stored in a $28^{\circ} \mathrm{C}$ incubator. After three days, the eggs hatched into neonates, which were shaken directly onto apples. These infested apples were put in a clean, dry plastic tray located in a cage with good air exchange. The cage was then placed in an incubator with a constant temperature of $28^{\circ} \mathrm{C}$. Another cage with a tray containing intact apples (i.e., without neonate inoculation) was stored in the same incubator. After five days, the infested and intact apples were both scanned for AE signal collection. The number of apples subjected to AE signal collection was 80 , including 30 intact apples and 50 with $\mathrm{CM}$ larvae infestation.

The larvae-infested apples were verified by cutting them open and examining them for larvae activity (i.e., live larvae, holes drilled by larvae, and microbial contamination brought by larvae). Before cutting them open, the infested apples had been scanned for AE signal collection to ascertain their infestation. The infested apples were not just apples with live larvae inside but also included apples with the above signs of infestation. For postharvest processing, apples that have been damaged by CM larvae cannot be sold as intact apples. Intact apples are apples with intact surfaces and inside structures, no bruises, no microbial contamination, and no larvae activity.

\section{Acoustic Emission Signal Collection}

Several views of the AE system used in this study are shown in figure 2 . The system sits in a room with a concrete padded floor built on a $20 \mathrm{~cm}$ of gravel above the loam soil bed. On the concrete pad is a table that holds a $15 \mathrm{~cm}$ layer of sand, topped with a $5 \mathrm{~cm}$ slab of granite. Insulated environmental chambers are set on the granite slab. The two black chambers are insulated with $2.5 \mathrm{~cm}$ black foam and sit on a rubber mat. The system was designed to reduce extraneous noises coming through the ground, wall, and room air into the environmental chamber.

The AE system is composed of sensors, a preamplifier, an I/O board, and signal processing software. As shown in figure 2, each apple was fixed to a piezoelectric sensor, and the door of the chamber was closed before signal acquisition. The acoustic sensor was attached to a preamplifier (model 1220A, Physical Acoustics Corp., Princeton Junction, N.J.), which was installed in a compartment within the data collection chamber. The AE data were collected and analyzed using AEwin software (PAC, 2003). The AE sensors and software were developed by a local company in Lexington, Kentucky. The sensor (R6 $\alpha$-SNAD 52) sensitivity ranged between 35 and $100 \mathrm{kHz}$. The sampling rate was 1 million samples $\mathrm{s}^{-1}$, and the record length was $100.25 \mathrm{~ms}$. An R6 sensor was mounted on the inner wall of each compartment and was used as a guard sensor. The R6 sensor was attached to a preamplifier (model 1220A, Physical Acoustics Corp.), which was set at 40 decibels $(\mathrm{dB})$ to reduce the effects of noise. The preamplifier transferred the collected data to the I/O board (PCI-2, Physical Acoustics Corp.) within the computer.

\section{AE Parameters}

The AEwin software extracted nine time domain features,
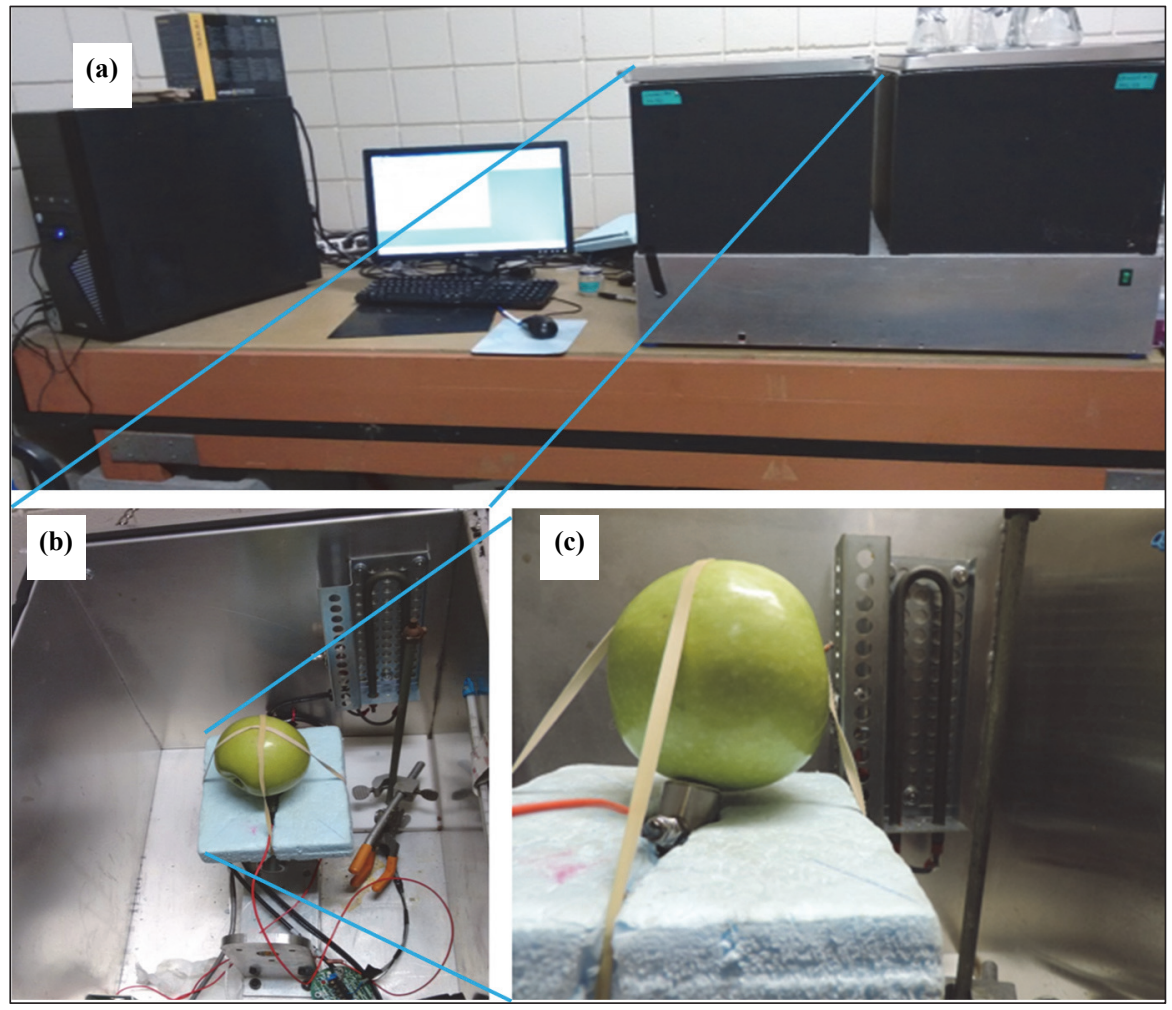

Figure 2. AE system: (a) outside view of system, (b) inside view of environmental chamber, and (c) apple in contact with sensor head. 
including risetime, counts, energy, duration, amplitude, ASL (average signal level), RMS (root mean square of signal), signal strength, and absolute energy. Figure 3 shows the features extractable from the AE signals. The signal was called an event every time a sound waveform reached the acoustic sensor. For every event, the waveform components above a threshold level were extracted and recorded as an AE hit. The acoustic sensor threshold level was always between 18 and $26 \mathrm{~dB}$ to eliminate background and environmental noise. From preliminary experiments, we determined that a threshold of $21 \mathrm{~dB}$ could eliminate most background noise. With a $21 \mathrm{~dB}$ threshold, the recorded background was approximately two hits per second. Each apple was fixed to the sensor, and the signal was recorded for $60 \mathrm{~min}$ for each apple. Signals for all apples were collected every day for four days. The risetime is the time from when an AE signal first crosses the threshold to its peak. Counts is the number of AE signal excursions over the detection threshold within a hit. The energy is a two-byte parameter derived from the integral of the rectified voltage signal over the duration of the AE hit. Duration is the time from the first threshold crossing to the end of the last threshold crossing of the AE signal. The amplitude is the maximum $\mathrm{AE}$ signal excursion (in $\mathrm{mV}$ ) during a hit. The amplitude is calculated using equation 1 :

$$
\begin{aligned}
\mathrm{dB} & =20 \times \log \left(V_{\max } / 1 \text { in } \mu \mathrm{V}\right) \\
& -(\text { preamplifier gain in } \mathrm{dB})
\end{aligned}
$$

where $V_{\max }$ is the peak signal voltage (in $\mathrm{mV}$ ) referred to the preamplifier input.

ASL is a measure of the continuously varying and average amplitude of the AE signal. RMS is a measure of the continuously varying $\mathrm{AE}$ signal voltage into the $\mathrm{AE}$ system. The signal strength (in attojoules, aJ) is the integral of the rectified voltage signal over the duration of the $\mathrm{AE}$ wave- form packet. Absolute energy (in aJ) is derived from the integral of the squared voltage signal divided by the reference resistance $(10 \mathrm{k} \Omega)$ over the duration of the $\mathrm{AE}$ waveform packet.

The software is capable of performing real-time fast Fourier transform (FFT) from an AE hit to get two frequency domains: peak frequency and frequency centroid. Therefore, a total of eleven features are generated by the AEwin software (PAC, 2003). Peak frequency is the frequency (in $\mathrm{kHz}$ ) at the point where the largest peak magnitude occurs during the record time length, and the frequency centroid (in $\mathrm{kHz}$ ) is calculated with equation 2 :

$$
\text { Frequency centroid }=\frac{\text { Sum }(\text { magnitude } \times \text { frequency })}{\text { Sum }(\text { magnitude })}
$$

Detailed descriptions of the features can be found in the PCI-2 manual (PAC, 2003). Typical signals for an infested apple and an intact apple are shown in figure 4.

\section{Data Analysis}

The recorded data included curves for the eleven features changing with time for a total of $60 \mathrm{~min}$. While $60 \mathrm{~min}$ is not feasible for quick on-line sorting of apples in postharvest processing, it was necessary to consider a duration that would give the best classification. Different signaling times were selected for analysis, which were in minutes $(5,15$, and $60 \mathrm{~min})$ and in seconds $(0.5,1,5,10,30,60$, and $120 \mathrm{~s})$. The signaling frequency was $0.5 \mathrm{~s}$ for all signaling times, implying that signals were collected every $0.5 \mathrm{~s}$. The first $0.5 \mathrm{~s}$ was considered noise and was therefore eliminated. The area under the curves was calculated using the trapezoid rule ("trapz" function in MATLAB R2014a, The MathWorks, Natick, Mass.). The curve for each feature was collapsed into a single value (area) for each feature. The single values for

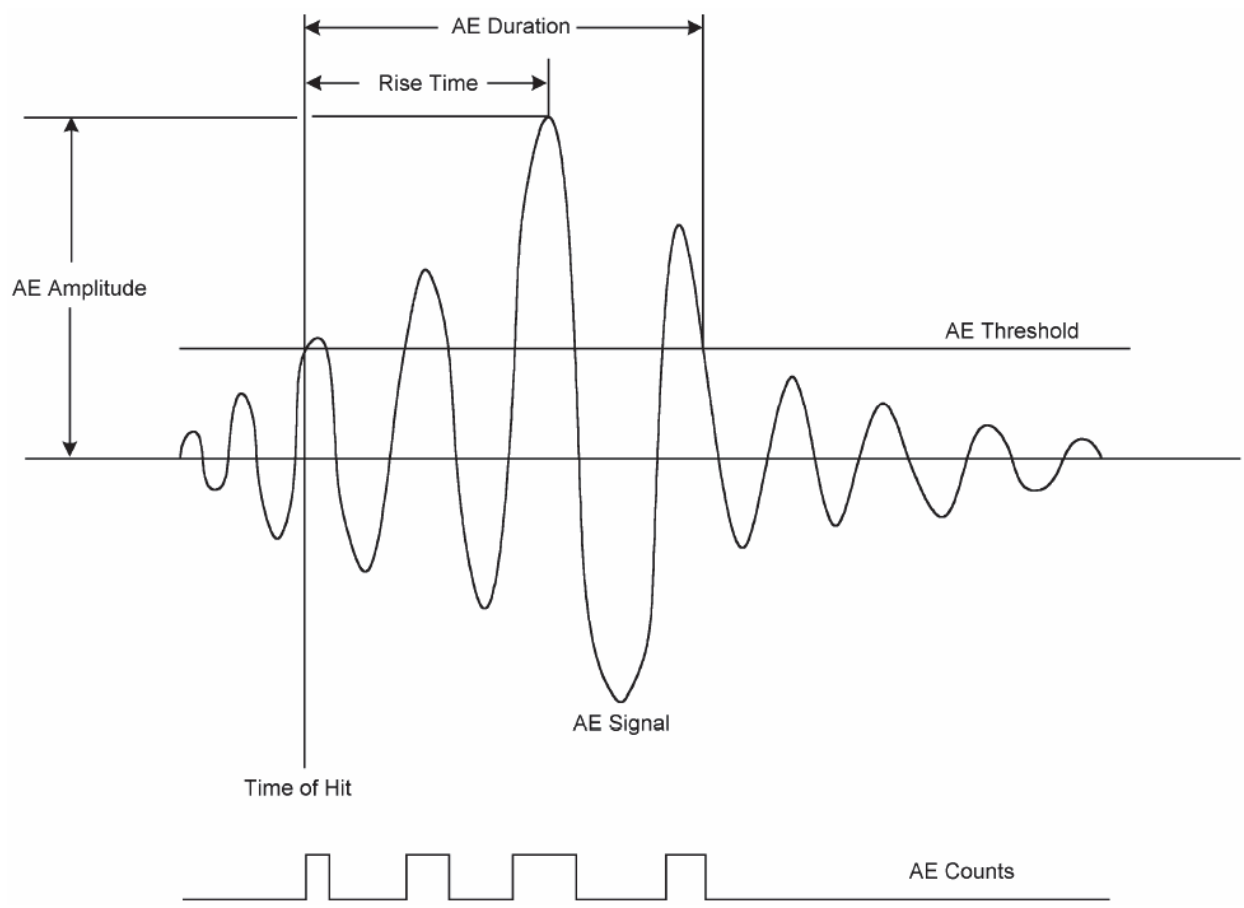

Figure 3. AE feature extraction diagram (PAC, 2003). 


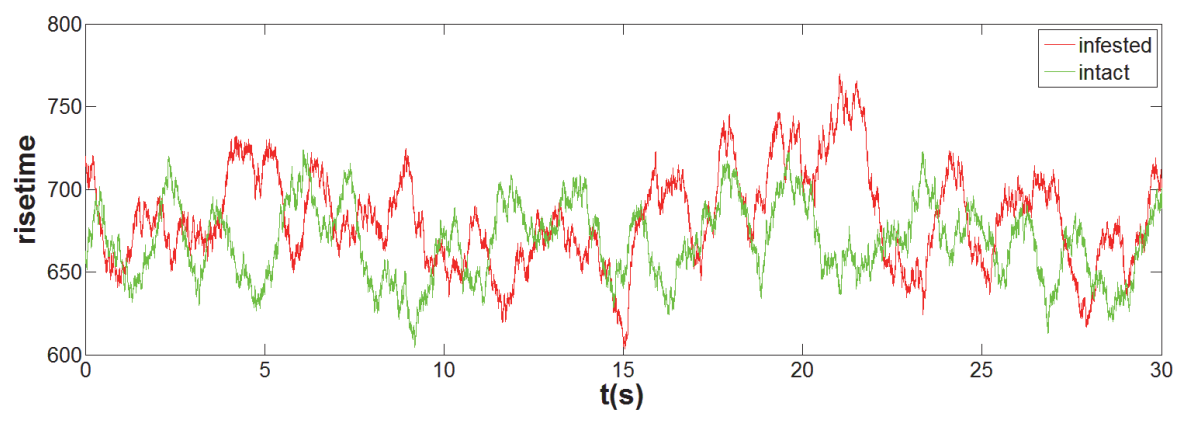

Figure 4. Sample AE signals (risetime changing with time in 30 s).

Table 1. Dataset for apple AE signal curve area for $1 \mathrm{~s}$ signaling time.

\begin{tabular}{|c|c|c|c|c|c|c|c|c|c|c|c|}
\hline Group $^{[\mathrm{a}]}$ & Risetime & Counts & Energy & Duration & Amplitude & ASL & RMS & $\begin{array}{c}\text { Signal } \\
\text { Strength }\end{array}$ & $\begin{array}{c}\text { Absolute } \\
\text { Energy }\end{array}$ & $\begin{array}{c}\text { Frequency } \\
\text { Centroid }\end{array}$ & $\begin{array}{c}\text { Peak } \\
\text { Frequency }\end{array}$ \\
\hline \multirow[t]{4}{*}{ Intact } & 767.18 & 2.51 & 0.07 & 1314.25 & 22.40 & 12.72 & 0.00 & 2346.75 & 1.72 & 214.58 & 5.76 \\
\hline & 641.80 & 2.27 & 0.05 & 1017.10 & 22.36 & 12.47 & 0.00 & 1820.05 & 17.54 & 214.73 & 6.82 \\
\hline & 624.62 & 2.28 & 0.02 & 928.44 & 22.40 & 12.45 & 0.00 & 1671.41 & 1.48 & 213.75 & 4.83 \\
\hline & 699.39 & 2.46 & 0.06 & 1205.04 & 22.41 & 12.54 & 0.00 & 2144.98 & 1.61 & 215.28 & 4.87 \\
\hline \multirow[t]{5}{*}{ Infested } & 705.46 & 2.94 & 0.06 & 1281.76 & 22.63 & 13.32 & 0.01 & 2328.61 & 1.86 & 214.82 & 4.21 \\
\hline & 812.80 & 2.77 & 0.07 & 1242.84 & 22.50 & 13.56 & 0.01 & 2265.65 & 1.79 & 214.72 & 3.62 \\
\hline & 625.89 & 2.54 & 0.04 & 1038.26 & 22.53 & 12.79 & 0.00 & 1874.50 & 1.63 & 215.15 & 4.87 \\
\hline & 757.34 & 2.43 & 0.05 & 1122.73 & 22.62 & 13.50 & 0.01 & 2011.00 & 1.62 & 215.91 & 4.29 \\
\hline & 705.46 & 2.94 & 0.06 & 1281.76 & 22.63 & 13.32 & 0.01 & 2328.61 & 1.86 & 214.82 & 4.21 \\
\hline
\end{tabular}

[a] Intact apples included 30 samples, and infested apples included 50 samples.

the eleven features of 80 samples were used as independent variables in the machine learning models. A sample dataset is shown in table 1 .

The machine learning methods included LDA and adaptive boosting, which were run using R software. The classification rate was used to assess the model's performance. Data were standardized and grouped into a training set (56 samples, $70 \%$ ) and a test set (24 samples, 30\%) using the "scale" and "createDataPartition" functions in the "caret" package in R (Williams, 2009). The full dataset and datasets with selected variables were analyzed with LDA and boosting using the "lda" (Teeling et al., 2005) and "boosting" (Alfaro et al., 2013) functions in the "mass" package in R, respectively. Feature ranking by importance was performed with a receiver operating characteristic (ROC) analysis using the "varImp" function in the "caret" package. Feature selection was performed by recursive feature elimination using the "rfecontrol" function in the "caret" package (Tsiliki et al., 2015).

\section{RESULTS AND DISCUSSION}

The relationship of acoustic signals to the quality of agrifood products was reviewed by Aboonajmi et al. (2015). Multiple features from acoustic signals with certain signaling times have been used to achieve good prediction of quality attributes and classify food materials into different categories. A similar approach was used in this study. For signaling times in minutes $(5,15,30$, and $60 \mathrm{~min})$, the classification rate was $100 \%$ for both the training and test sets, either with selected features or all eleven features. A perfect sorting of infested apples from intact apples was achieved. Because long signaling times are not practical for on-line sorting applications, the results discussed here focus on signaling times in seconds $(0.5,1,5,10,60$, and $120 \mathrm{~s})$. Signal- ing times of 60 and $120 \mathrm{~s}$ are considered second-level signals because 1 or $2 \mathrm{~min}$ is considered a short timeframe. In addition, the use of multiple features in modeling will increase the required capacity and cost of data storage and computation. Selection of important features and elimination of redundant features can increase the efficiency of AE data analysis. Figure 5 shows an example of feature ranking by importance for $1 \mathrm{~s}$ signals. For signaling times in seconds $(0.5$, $1,5,10,60$, and $120 \mathrm{~s}$ ), the LDA and boosting classification rates for the full dataset and for selected variables are shown in tables 2 and 3 , respectively.

Figure 5 shows amplitude to be the most important feature because amplitude represents the maximum value de-

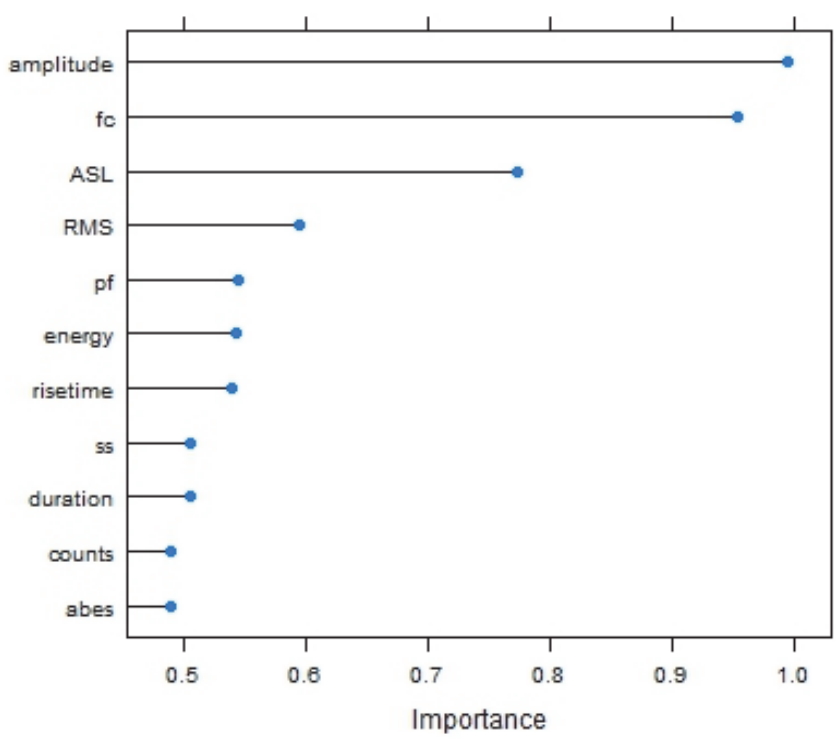

Figure 5. Sample feature ranking by importance for $1 \mathrm{~s}$ signals $(\mathrm{fc}=$ frequency centroid, $\mathrm{pf}=$ peak frequency, $\mathrm{ss}=$ signal strength, and abes $=$ absolute energy). 
rived from voltage during an AE hit. Therefore, amplitude is expected to be higher for infested apples than for intact apples. The other three features with coefficients greater than 0.6 are frequency centroid, ASL, and RMS, which are followed by peak frequency, energy, and risetime. The features shown in figure 5 are in accordance with other AE studies. Energy, counts, amplitude, frequency, and duration were used for classification of extruded bread with different water activities (Swietlicka et al., 2015). Amplitude and sound intensity were used to predict carrot firmness (Liu et al., 2016). Several attributes of acoustic events (i.e., average amplitude of the acoustic signal, average time, and average energy of the acoustic event) were used to assess apple tissue quality (Zdunek and Bednarczyk, 2006). Wiktor et al. (2016) used destructive AE testing to assess the electrical conductivity of apple slices after applying a pulsed electrical field. They found that the electrical conductivity correlated well with the number of acoustic events, the energy of the acoustic event, and the amplitude. Roversi and Piazza (2016) showed that acoustic analysis can highlight two main phenomena: sound propagation and the cracking events generating the sound. These two phenomena were correlated with signal amplitude and the number of acoustic events, respectively, according to the research by Zdunek et al. (2011). Similarly, apple crispness and crunchiness were evaluated by AE events and mean AE amplitude (Zdunek et al., 2010). RMS was found to be a good predictor for leak-testing of metals and construction materials (PAC, 2003). In the cores of the infested apples, the holes drilled by CM larvae behaved as leaks. The features used as important classifiers in this study are similar to the features used in the studies listed above. The number of features used in this study is higher than in most previous research and can be used as a reference for future studies to test more features and find the most important features.

Classification rates of the full dataset for signaling times in seconds $(0.5,1,5,10,30,60$, and $120 \mathrm{~s})$ are shown in table 2 . The classification rates for the signaling times in seconds varied from $67 \%$ to $100 \%$ for the training set and from $57 \%$ to $100 \%$ for the test set for LDA. The classification rate obtained for the boosting method was greater than $86 \%$, which means that the model provided a better fit for areas calculated from the AE signals. The success of adaptive boosting shows the potential of the AE system for sorting of $\mathrm{CM}$-infested apples. There was no consistent trend showing that the classification rate decreased with reduced signaling time, which may be because the number of samples (80) was not large enough to reveal the differences between infested apples and intact apples. A larger dataset usually generates better classification (Dai et al., 2015; Sharma and Paliwal, 2015). For signaling times from 10 to $0.5 \mathrm{~s}$, the classification

Table 2. Classification rates (\%) using the full data set.

\begin{tabular}{cccccc}
\hline Signaling & \multicolumn{2}{c}{ LDA } & & \multicolumn{2}{c}{ Boosting } \\
\cline { 2 - 3 } \cline { 5 - 6 }$(\mathrm{s})$ & Training & Test & & Training & Test \\
Set & 80 & Set & & Set & Set \\
\hline 120 & 100 & 74 & & 100 & 96 \\
60 & 67 & 100 & & 100 & 100 \\
30 & 100 & 96 & & 100 & 100 \\
10 & 100 & 100 & & 100 & 100 \\
5 & 94 & 91 & & 100 & 100 \\
1 & 91 & 83 & & 100 & 87 \\
0.5 & & &
\end{tabular}

Table 3. Classification rates (\%) using selected features.

\begin{tabular}{ccccccc}
\hline \multirow{2}{*}{$\begin{array}{c}\text { Signaling } \\
\text { Time }\end{array}$} & & \multicolumn{2}{c}{ LDA } & & \multicolumn{2}{c}{ Boosting } \\
\cline { 3 - 4 }$(\mathrm{s})$ & Features Eliminated & Set & Set & & Set & Set \\
\hline 120 & Duration & 75 & 74 & & 95 & 90 \\
60 & Risetime, counts & 100 & 100 & & 100 & 100 \\
30 & Peak frequency, energy & 67 & 57 & & 100 & 100 \\
10 & Energy, risetime & 100 & 96 & & 100 & 100 \\
5 & Signal strength, risetime & 100 & 100 & & 100 & 100 \\
1 & Absolute energy, counts & 94 & 91 & & 100 & 100 \\
0.5 & Energy, signal strength & 91 & 78 & & 100 & 78 \\
\hline
\end{tabular}

rates for LDA decreased with signaling time for both the training and test sets. However, when adaptive boosting was used, the classification rate was perfect $(100 \%)$ for the same signaling times, apart from the test with $0.5 \mathrm{~s}$ sampling time, which showed a decrease. Adaptive boosting showed better performance than LDA because it is an ensemble method. A few nondestructive methods have been used for successful detection of CM-infested apples, including different $\mathrm{x}$-ray systems (Hansen et al., 2005; Jiang et al., 2008). Early detection of CM is difficulty in X-ray images (Haff and Toyofuku, 2008). Early-stage CM-infested apple detection was not a problem in our study because five-day larvae infestation was used. Another study using hyperspectral imaging and multivariate analysis achieved $86 \%$ classification (Rady et al., 2017). However, for both x-ray and hyperspectral imaging, practical application would be difficult because of the implementation costs and sensitivity of the methods due to the large amount of image data.

For the results shown in table 3, feature selection was performed first, and then LDA and boosting were performed with the selected features. A majority of the features were selected, and only the eliminated features are listed in table 3. There was no consistent trend in the eliminated features for different signaling times, which may have been caused by variations in insect activity. It is possible that CM larvae moved or fed only at certain times during the different signaling times. Comparing the classification rates in table 3 with those in table 2, most of the classification rates remained the same, while some became worse. Because the feature selection in this study made classification performance worse for some of the signaling times, feature selection is not recommended.

\section{CONCLUSION}

Based on the AE signals collected and analyzed from CM-infested and intact apples over a 60 min signaling time, we can conclude that $\mathrm{AE}$ has potential for use as a nondestructive method for early detection of CM infestation in apples. Perfect classification rates of $100 \%$ were obtained for apples using AE signals collected for more 5 min of signaling time, irrespective of the machine learning method (i.e., LDA or boosting). For signals collected for less than $5 \mathrm{~min}$, there was no consistent trend in classification rates for the LDA model. The model built with adaptive boosting showed perfect classification rates for signaling times up to $1 \mathrm{~s}$ duration, apart from the test with $0.5 \mathrm{~s}$ sampling time, for which the classification rate was $87 \%$. The expectation is that more improvement will be observed in the classification rates, 
even at $0.5 \mathrm{~s}$, when more samples are used for building the predictive model.

\section{ACKNOWLEDGEMENTS}

The information reported in this paper (\#:17-05-035) is a project of the Kentucky Agricultural Experiment Station and it is published with the approval of the Director. This work was supported by the USDA National Institute of Food and Agriculture (Multistate Project No. 1007893). We also acknowledge Dr. John Stencel (Tribo Flow Separations LLC, Lexington, Ky.), Dr. Clair Hicks (Department of Animal and Food Science, University of Kentucky), and Dr. Fred Payne (Department of Biosystems and Agricultural Engineering, University of Kentucky) for their contributions to the development of the AE system used for this project.

\section{REFERENCES}

Aboonajmi, M., Jahangiri, M., \& Hassan-Beygi, S. R. (2015). A review on application of acoustic analysis in quality evaluation of agro-food products. J. Food Proc. Preserv., 39(6), 31753188. https://doi.org/10.1111/jfpp.12444

Alfaro, E., Gamez, M., \& Garcia, N. (2013). Adabag: An R package for classification with boosting and bagging. J. Stat. Software, 54(2), 1-35. https://doi.org/10.18637/jss.v054.i02

Dai, Q., Cheng, J.-H., Sun, D.-W., Pu, H., Zeng, X.-A., \& Xiong, Z. (2015). Potential of visible/near-infrared hyperspectral imaging for rapid detection of freshness in unfrozen and frozen prawns. J. Food Eng., 149, 97-104. https://doi.org/10.1016/j.jfoodeng.2014.10.001

Dorn, S., \& Piñero, J. C. (2009). How do key tree-fruit pests detect and colonize their hosts: Mechanisms and application for IPM. In Biorational tree-fruit pest management (pp. 85-109). Wallingford, UK: CABI. https://doi.org/10.1079/9781845934842.0085

Ghosh, D., Stencel, J. M., Hicks, C. D., Payne, F., \& Ozevin, D. (2013). Acoustic emission signal of Lactococcus lactis before and after inhibition with $\mathrm{NaN}_{3}$ and infection with bacteriophage c2. ISRN Microbiol., 2013, article 257313. https://doi.org/10.1155/2013/257313

Gorji-Chakespari, A., Nikbakht, A. M., Sefidkon, F., GhasemiVarnamkhasti, M., Brezmes, J., \& Llobet, E. (2016). Performance comparison of fuzzy ARTMAP and LDA in qualitative classification of Iranian Rosa damascena essential oils by an electronic nose. Sensors, 16(5), 636. https://doi.org/10.3390/s16050636

Haff, R. P., \& Toyofuku, N. (2008). X-ray detection of defects and contaminants in the food industry. Sensing Instrum. Food Qual. Saf., 2(4), 262-273. https://doi.org/10.1007/s11694-008-9059-8

Hansen, J. D., Schlaman, D. W., Haff, R. P., \& Yee, W. L. (2005). Potential postharvest use of radiography to detect internal pests in deciduous tree fruits. J. Entomol. Sci., 40(3), 255-262. https://doi.org/10.18474/0749-8004-40.3.255

Ivanovic, L., \& Jelocnik, M. (2017). Analysis and planning of apple production as factor of rural development support. Econ. Anal., 42(3-4), 78-85.

Jiang, J.-A., Chang, H.-Y., Wu, K.-H., Ouyang, C.-S., Yang, M.M., Yang, E.-C., ... Lin, T.-T. (2008). An adaptive image segmentation algorithm for $\mathrm{x}$-ray quarantine inspection of selected fruits. Comput. Electron. Agric., 60(2), 190-200. https://doi.org/10.1016/j.compag.2007.08.006

Konopacka, D., Jesionkowska, K., Kruczynska, D., Stehr, R., Schoorl, F., Buehler, A., ... Holler, I. (2010). Apple and peach consumption habits across European countries. Appetite, 55(3),
478-483. https://doi.org/10.1016/j.appet.2010.08.011

Li, H., Chen, Q., Zhao, J., \& Wu, M. (2015). Nondestructive detection of total volatile basic nitrogen (TVB-N) content in pork meat by integrating hyperspectral imaging and colorimetric sensor combined with a nonlinear data fusion. LWT - Food Sci. Tech., 63(1), 268-274. https://doi.org/10.1016/j.lwt.2015.03.052

Liu, Y., Sun, Y. H., Yang, L., Yu, L. B., Mao, Q., Hou, J. M., ... Liu, C. (2016). Relationship between carrot firmness with acoustic signal characteristics. J. Food Proc. Eng., 49(2), e12384. https://doi.org/10.1111/jfpe.12384

Mao, J., Yu, Y., Rao, X., \& Wang, J. (2016). Firmness prediction and modeling by optimizing acoustic device for watermelons. $J$. Food Eng., 168, 1-6. https://doi.org/10.1016/j.jfoodeng.2015.07.009

Milczarek, R. R., Saltveit, M. E., Garvey, T. C., \& McCarthy, M. J. (2009). Assessment of tomato pericarp mechanical damage using multivariate analysis of magnetic resonance images. Postharvest Biol. Tech., 52(2), 189-195. https://doi.org/10.1016/j.postharvbio.2009.01.002

Mohana, S. H., Prabhakar, C. J., \& Praveen Kumar, P. U. (2013). Surface defect detection and grading of apples. In Multimedia processing, communication, and infomational technology 2013 (pp. 57-64). Red Hook, NY: Curran Associates.

Mohareb, F., Papadopoulou, O., Panagou, E., Nychas, G.-J., \& Bessant, C. (2016). Ensemble-based support vector machine classifiers as an efficient tool for quality assessment of beef fillets from electronic nose data. Anal. Methods, 8(18), 37113721. https://doi.org/10.1039/C6AY00147E

Muravin, B. (2009). Acoustic emission science and technology. Journal of building and infrastructure engineering of the Israeli association of engineers and architects. Retrieved from http://www.muravin.com/downloads/Muravin\%20\%20Acoustic\%20Emission\%20Science\%20and\%20Technology .pdf

PAC. (2003). PCI-2 based AE system user's manual. Rev.1a. Princeton, NJ: Physical Acoustics Corporation.

Rady, A., Ekramirad, N., Adedeji, A. A., Li, M., \& Alimardani, R. (2017). Hyperspectral imaging for detection of codling moth infestation in GoldRush apples. Postharvest Biol. Tech., 129, 37 44. https://doi.org/10.1016/j.postharvbio.2017.03.007

Roversi, T., \& Piazza, L. (2016). Changes in minimally processed apple tissue with storage time and temperature: Mechanicalacoustic analysis and rheological investigation. European Food Res. Tech., 242(3), 421-429. https://doi.org/10.1007/s00217015-2553-4

Schatzki, T. F., Haff, R. P., Young, R., Can, I., Le, L. C., \& Toyofuku, N. (1997). Defect detection in apples by means of Xray imaging. Trans. ASAE, 40(5), 1407-1415. https://doi.org/10.13031/2013.21367

Sharma, A., \& Paliwal, K. K. (2015). Linear discriminant analysis for the small sample size problem: An overview. Intl. J. Machine Learn, Cybernet, 6(3), 443-454. https://doi.org/10.1007/s13042-013-0226-9

Stencel, J. M., Song, H., \& Cangialosi, F. (2009). Automated foam index test: Quantifying air entraining agent addition and interactions with fly ash-cement admixtures. Cement Concrete Res., 39(4), 362-370. https://doi.org/10.1016/j.cemconres.2009.01.010

Swietlicka, I., Muszynski, S., \& Marzec, A. (2015). Extruded bread classification on the basis of acoustic emission signal with application of artificial neural networks. Intl. Agrophysics, 29(2), 221-229. https://doi.org/10.1515/intag-2015-0022

Teeling, E. C., Springer, M. S., Madsen, O., Bates, P., O’Brien, S. J., \& Murphy, W. J. (2005). A molecular phylogeny for bats illuminates biogeography and the fossil record. Science, 307(5709), 580-584. https://doi.org/10.1126/science.1105113 
Tsiliki, G., Munteanu, C. R., Seoane, J. A., Fernandez-Lozano, C., Sarimveis, H., \& Willighagen, E. L. (2015). RRegrs: An R package for computer-aided model selection with multiple regression models. J. Cheminfo., 7(1), 46. https://doi.org/10.1186/s13321-015-0094-2

Walker, J. T., Lo, P. L., Horner, R. M., Park, N. M., Hughes, J. G., \& Fraser, T. M. (2013). Codling moth (Cydia pomonella) mating disruption outcomes in apple orchards. New Zealand Plant Prot., 66, 259-263.

Wiktor, A., Gondek, E., Jakubczyk, E., Nowacka, M., Dadan, M., Fijalkowska, A., \& Witrowa-Rajchert, D. (2016). Acoustic emission as a tool to assess the changes induced by pulsed electric field in apple tissue. Innov. Food Sci. Emerg. Tech., 37(part C), 375-383. https://doi.org/10.1016/j.ifset.2016.04.008

Williams, G. J. (2009). Rattle: A data mining GUI for R. $R$ Journal, $1(2), 45-55$

Xu, L., Yu, X., Liu, L., \& Zhang, R. (2016). A novel method for qualitative analysis of edible oil oxidation using an electronic nose. Food Chem., 202, 229-235. https://doi.org/10.1016/j.foodchem.2016.01.144

Yang, S. F., Xue, L., \& Zhao, J. M. (2014). Detecting system of crop disease stress based on acoustic emission and virtual technology. In Applied mechanics and materials (Vol. 556, pp. 3331-3334). Zurich, Switzerland: Trans Tech Publications.

Zdunek, A., \& Bednarczyk, J. (2006). Effect of mannitol treatment on ultrasound emission during texture profile analysis of potato and apple tissue. J. Texture Studies, 37(3), 339-359. https://doi.org/10.1111/j.1745-4603.2006.00055.x

Zdunek, A., Cybulska, J., Konopacka, D., \& Rutkowski, K. (2011). Evaluation of apple texture with contact acoustic emission detector: A study on performance of calibration models. J. Food Eng., 106(1), 80-87.

https://doi.org/10.1016/j.jfoodeng.2011.04.011

Zdunek, A., Konopacka, D., \& Jesionkowska, K. (2010). Crispness and crunchiness judgment of apples based on contact acoustic emission. J. Texture Studies, 41(1), 75-91. https://doi.org/10.1111/j.1745-4603.2009.00214.x

Zhang, L., \& McCarthy, M. J. (2012). Measurement and evaluation of tomato maturity using magnetic resonance imaging. Postharvest Biol. Tech., 67, 37-43. https://doi.org/10.1016/j.postharvbio.2011.12.004 